\title{
Therapeutic Effects of Bee Venom on Immunological and Neurological Diseases
}

\author{
Deok-Sang Hwang ${ }^{1, \dagger}$, Sun Kwang Kim ${ }^{2, \dagger}$ and Hyunsu Bae ${ }^{2, *}$
}

1 Department of Korean Medicine Obstetrics and Gynecology, College of Korean Medicine, Kyung Hee University, 26 Kyungheedae-ro, Dongdaemun-gu, Seoul 130-701, Korea; E-Mail: soulhus@gmail.com

2 Department of Physiology, College of Korean Medicine, Kyung Hee University, 26 Kyungheedae-ro, Dongdaemun-gu, Seoul 130-701, Korea; E-Mail: skkim77@khu.ac.kr

$\dagger$ These authors contributed equally to this work.

* Author to whom correspondence should be addressed; E-Mail: hbae@khu.ac.kr; Tel.: +82-2-961-9316; Fax: +82-70-4194-9316.

Academic Editor: Lai Ren

Received: 15 May 2015 / Accepted: 24 June 2015 / Published: 29 June 2015

\begin{abstract}
Bee Venom (BV) has long been used in Korea to relieve pain symptoms and to treat inflammatory diseases, such as rheumatoid arthritis. The underlying mechanisms of the anti-inflammatory and analgesic actions of BV have been proved to some extent. Additionally, recent clinical and experimental studies have demonstrated that BV and BV-derived active components are applicable to a wide range of immunological and neurodegenerative diseases, including autoimmune diseases and Parkinson's disease. These effects of BV are known to be mediated by modulating immune cells in the periphery, and glial cells and neurons in the central nervous system. This review will introduce the scientific evidence of the therapeutic effects of BV and its components on several immunological and neurological diseases, and describe their detailed mechanisms involved in regulating various immune responses and pathological changes in glia and neurons.
\end{abstract}

Keywords: Bee Venom; immunological diseases; neurological diseases 


\section{Introduction}

Bee Venom (BV) therapy is a form of medicine originated from the ancient Greece and China. Several scientific reports suggesting the anti-rheumatic and anti-inflammatory effects of BV have been published for a hundred years [1,2]. In Korea, BV has long been used to relieve pain and to treat various diseases, such as arthritis, rheumatism, herniation nucleus pulpous, cancer, asthma, and skin diseases [3-5]. It is administered systemically or in the form of chemical stimulation of acupoints, so called "BV acupuncture" or "apipuncture". BV is known to contain many active components, including peptides (e.g., melittin and apamin), enzymes (e.g., phospholipase A2 (PLA2)), and small molecules (e.g., histamine). Recent studies suggested further that BV and BV-derived active components might have potent therapeutic effects on refractory immunological and neurodegenerative diseases including allergic disorders, autoimmune diseases, amyotrophic lateral sclerosis (ALS), and Parkinson's disease (PD) [3,6-9], however well-controlled, randomized clinical studies are still insufficient.

In this review, the underlying mechanisms of BV-induced regulation of immune responses as well as of neuronal and glial pathology in refractory immunological and neurological diseases will be discussed, based mainly on the articles that have been published in the last decade. In addition, the therapeutic effects and mechanisms of BV-derived active components, especially focusing on $\mathrm{PLA}_{2}$, melittin and apamin will be introduced. Finally, we will comment on the future perspectives in the research area of BV therapy.

\section{Therapeutic Effects of Bee Venom on Immunological Diseases}

\subsection{Effects on Allergic Disorders}

The initial event responsible for the development of allergic disorders, such as asthma, allergic rhinoconjunctivitis, and atopic eczema, is the generation of allergen-specific $\mathrm{CD}^{+} \mathrm{T}$ cells [10]. In a general view, allergy is a T helper 2 ( Th2) cells-mediated disease that involves the hyperproduction of specific immunoglobulin E (IgE) antibodies to which interleukin-4 (IL-4) and IL-13, the key Th2-specific cytokines, mainly contribute [11].

BV therapy is a kind of allergen-specific immunotherapy (SIT) that has been carried out for a long time. Although the mechanism of SIT remains poorly understood, hitherto several features, including modifications of antigen presenting cells (APCs), T cells, and B cells, as well as both the number and the function of effector cells that mediate the allergic response have been clarified [12]. In clinical trials, it was reported that SIT increases the production of IL-10 by APCs, including B cells, monocytes, and macrophages [12]. The efficacy of SIT has been emphasized in insect venom allergy and respiratory allergies. BV immunotherapy has early and late influences on major cells of allergic inflammation [10]. Venom immunotherapy induces a monocyte activation characterized by a delayed overproduction of IL-12 and tumor necrosis factor alpha (TNF- $\alpha$ ), which are cytokines related to the inhibition of Th2 cells [13]. BV immunotherapy is known to generate IL-10 and transforming growth factor beta (TGF- $\beta$ ), which potently suppresses IgE production and increases IgG4 and IgA, simultaneously [14]. Our previous study demonstrated that BV induces Th1 lineage development from $\mathrm{CD}^{+} \mathrm{T}$ cells without affecting Th2 cells, by increasing the expression of a Th1-specific cytokine, interferon gamma (INF- $\gamma$ ), via an upregulation of Th1-specific transcription factor, T-bet [15]. 
$\mathrm{CD}^{+} \mathrm{CD} 25^{+} \mathrm{Foxp}^{+}$regulatory $\mathrm{T}$ cells (Tregs) play a pivotal role in the maintenance of tolerance in the immune system and are involved in the control of transplantation tolerance, tumor immunity, allergy, and infection [16,17]. Tregs could regulate allergic disorders through several inhibitory pathways, including suppression of Th2 immune responses, of Th17 cells, and of T cell migration to tissues [11]. It has been suggested that an essential step in successful BV immunotherapy is associated with the presence of Tregs, which secrete IL-10, consequently inhibiting the secretion of cytokines IL-4, IL-5, and IL-13 from Th2 cells, in turn impeding specific IgE production [18]. For example, our recent study showed that BV treatment increased Treg populations, augmented the production of IL-10, and suppressed the production of Th1, Th2, and Th17-related cytokines, resulting in bronchial inflammation with a reduction in the degranulation of mast cells and eosinophils in an OVA-induced allergic asthma murine model [3]. Taken together, the immunological mechanisms of BV immunotherapy include a shift toward Th1 cytokines, an increase in the number of peripheral Tregs, and an upregulation of different markers expressed on $\mathrm{CD}^{+} \mathrm{T}$ cells $[10,19]$.

The most important allergen in $\mathrm{BV}$ is $\mathrm{PLA}_{2}$, which induces rapid leukotriene $\mathrm{C} 4$ production from purified human basophils within $5 \mathrm{~min}$, while IL-4 expression and production is induced at later time-points without histamine release [20]. Direct injection of the BV-derived PLA2 (bvPLA2) into inguinal lymph nodes enhanced allergen-specific $\mathrm{IgG}$ and $\mathrm{T}$-cell responses and stimulated the production of the Th1-dependent subclass IgG2a [21]. Melittin, a major peptide component of BV, is reported to trigger lysis of a mast cells, which can lead to the release of histamine and other intracellular components into surrounding tissues [18]. In our unpublished data [22], bvPLA2 increased Treg population in vitro and in vivo more potently than $\mathrm{BV}$, resulting in prevention of ovalbumin-induced allergic asthma in mice with suppression of various effector cells, such as eosinophils, lymphocytes, and macrophages, and of Th2 cytokines and serum IgE.

\subsection{Effects on Autoimmune and Inflammatory Diseases}

Autoimmune diseases, such as rheumatoid arthritis, systemic lupus erythematosus, and multiple sclerosis, have been understood to be Th1-dominant diseases, however, the important roles of Th17 cells and Tregs in autoimmune diseases have recently emerged [23]. Rheumatoid arthritis is a common autoimmune disease, yet current conventional therapies are not always successful [24]. BV has been traditionally used to treat chronic inflammatory diseases, including rheumatoid arthritis [4]. Especially, the anti-rheumatic and anti-inflammatory effects of BV have been understood as of one hundred years ago [1,2]. Previous study has demonstrated that BV injection into the Zusanli acupoint has both anti-inflammatory and anti-nociceptive effects on Freund's adjuvant-induced arthritis in rats [25]. Effect of combined application of bee-venom therapy and medication is superior to the simple use of medication in relieving rheumatoid arthritis and might reduce the commonly-taken doses of Western medicines [26]. These anti-arthritis effects have been reported in several arthritis models, and these effects of BV might be associated with melittin, a major peptide component of BV, which has anti-inflammatory

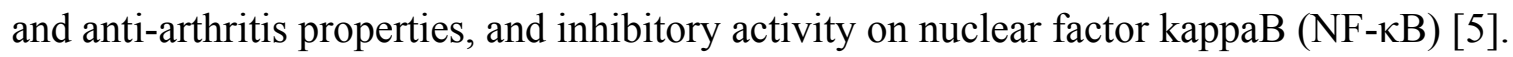

We previously examined the effects of BV on the nitric oxide (NO) generation by lipopolysaccharide (LPS) or sodium nitroprusside (SNP) in RAW264.7 macrophages, and the expression of inducible nitric oxide synthase (iNOS), cyclooxygenase 2 (COX-2), NF- $\mathrm{B}$ and mitogen-activated protein kinase 
(MAPK) with RT-PCR in LPS stimulated RAW 264.7 cells. The results showed that BV suppressed NO production and decreased the level of iNOS and COX-2 expression, possibly through suppressing NF- $\kappa \mathrm{B}$ and MAPK [27]. We also performed microarray analysis to evaluate the global gene expression profiles of macrophage cell treated with BV. We found that BV decreased the expression of various genes, including mitogen-activated protein kinase kinase kinase 8 (MAP3K8), TNF, suppressor of cytokine signaling 3 (SOCS3), TNF-receptor-associated factor 1 (TRAF1), JUN, and CREB binding protein (CBP), related to the inflammatory effects, which occur in LPS-treated RAW264.7 cells [28]. Other studies support these observations. For example, BV and melittin prevent LPS- or SNP-induced NO and prostaglandin E2 production via c-Jun N-terminal kinase (JNK) pathway dependent inhibition of NF- $\kappa$ B [29]. BV also suppressed adjuvant-induced arthritis in rats by targeting TNF- $\alpha$ and NF- $\mathrm{B}$ activation [30]. These findings indicate that BV may have anti-inflammatory effects in rheumatoid arthritis.

Lupus nephritis, a serious complication of systemic lupus erythematosus, is mediated by the glomerular inflammation involving the production of autoantibodies against the nucleus and of cytokines/chemokines, which ultimately results in irreversible renal damage [31,32]. New Zealand Black/White F1 female mice age-dependently develop autoimmune disease, which is characterized by glomerulonephritis, proteinuria, and renal dysfunction [33]. Using this animal model, we showed that BV treatment significantly delayed the development of proteinuria, prevented renal inflammation, reduced tubal damage, and decreased immune deposits in the glomeruli, and these results are closely associated with a BV-induced increase in splenic Tregs and decrease in renal proinflammatory cytokines, TNF- $\alpha$ and IL-6 [8]. These results suggest that BV therapy has the potential to modulate autoimmune response in lupus nephritis, possibly by enhancing Tregs and suppressing renal inflammation.

Multiple sclerosis is a chronic inflammatory disease of the central nervous system (CNS) that affects more than one million people worldwide. Its clinical symptoms include ataxia, loss of coordination, sensory impairment, cognitive dysfunction, and fatigue [34]. The pathogenesis of multiple sclerosis is known to be an autoimmune $\mathrm{T}$ cell responses, in which Th1 and Th17 cells are critically involved [35]. An animal model of experimental autoimmune encephalomyelitis (EAE) has been widely used for the study of multiple sclerosis, because clinical and pathological features of EAE are very similar to those of multiple sclerosis. We previously demonstrated that BV treatment has a neuroprotective effect against immune cell infiltration and Th1/Th17 differentiation via increasing Tregs in EAE mouse model [36]. Very recently, another research group has also reported that BV acupuncture attenuates the development and progression of EAE in rats by upregulating Tregs and suppressing Th1/Th17 cell responses [37]. These results suggest that BV has the potential to become a therapeutic agent for multiple sclerosis, and warrants further investigation of BV and bvPLA2 as potent modulators of autoimmune $\mathrm{T}$ cell responses in the CNS.

\section{Therapeutic Effects of Bee Venom on Neurological Diseases}

Parkinson's disease (PD) is one of the most common progressive neurodegenerative disorders, which is characterized clinically by bradykinesia, resting tremor, rigidity, and disturbances in posture and gait resulting from the selective, irreversible loss of dopaminergic (DA) neurons in the substantia nigra (SN) and their terminals in the striatum [38,39]. Activated microglia, innate immune cells in the CNS, near the degenerating DA neurons is known to be a key mediator of neuroinflammation in 
PD $[39,40]$. BV acupuncture was reported to be anti-inflammatory and anti-neurodegenerative, and to improve motor symptoms in PD clinical trials [6]. In a 1-methyl-4-phenyl-1,2,3,6-tetrahydropyridine (MPTP)-induced mouse model of PD, BV improved the survival percentage of tyrosine hydroxylase ${ }^{+}$ cells to $70 \%$ on day 1 and $78 \%$ on day 3 compared with normal mice, and reduced expression of the inflammation markers macrophage antigen complex-1 (MAC-1) and iNOS in the SN [41]. Our previous study also revealed that modulation of peripheral immune tolerance by Tregs may contribute to the neuroprotective effect of BV in the MPTP animal model of PD [42]. Recently, apamin, a specific component of BV, was also shown to have a protective effect in animal models of PD [43].

In an animal model of amyotrophic lateral sclerosis (ALS), mutant human superoxide dismutase 1 (hSOD1) transgenic mice, BV acupuncture inhibited microglia activation and phospho-p38 MAPK expression in the CNS, resulting in improvement of motor activity [9]. It also has been reported that melittin ameliorated the inflammation of lung and spleen in an ALS animal model [44]. BV might be helpful in reducing glutamatergic cell toxicity, which has been reported in many neurodegenerative diseases, including PD, Alzheimer's disease, and ALS, through the inhibition of MAP kinase activation (e.g., JNK, ERK, and p38) following exposure to glutamate [45]. Although scientific evidence is still limited in this research area, we strongly believe that the aforementioned results may lead to future advanced studies, elucidating the therapeutic effects of BV and its active components on various neurodegenerative diseases and its underlying mechanisms. Indeed, in several preliminary results, we found that $\mathrm{BV}$ and BV-derived active components ameliorate Alzheimer's disease, Parkinson's disease, and chronic neuropathic pain by modulating peripheral immune and inflammatory responses, as well as modulating central glial activation.

\section{Conclusions and Perspectives}

In this review, we introduced the therapeutic effects of $\mathrm{BV}$ and its major components on immunological and neurological diseases, and discussed its underlying mechanisms. We propose that $\mathrm{BV}$ is a strong immune modulator that may subsequently affect the CNS glia and neurons. BV also seems to play a role in maintaining homeostasis in our body's immune system and nervous system, because $\mathrm{BV}$ therapy can regulate two immunologically opposite conditions, i.e., allergic disorders (Th2 dominant) and autoimmune diseases (Th1 dominant). It remains to be understood how the same treatments of BV or BV-derived active components could modulate both conflicting diseases. Thus, other T cell populations, such as Th17 cells and Tregs, have emerged as a key players in BV-induced modulation of immune and nervous system. Th17 cells are known to play an important role in the pathogenesis of autoimmune, as well as allergic, diseases [46,47]. In contrast, Tregs inhibits activation of both Th1 and Th2 cells, and of Th17 cells, thereby suppressing autoimmune and allergic diseases $[11,35]$. Indeed, several recent studies reported that BV or bvPLA2 could upregulate peripheral Tregs and/or suppress Th17 responses in various animal models of both diseases [3,8,36,37,48]. Further studies on this issue might shed light on our understandings of such homeostatic therapeutic effects of BV.

In addition, it should be noted that BV is called a "double-edged sword" having nociceptive and anti-nociceptive effects [49], and BV itself could act as a strong allergen. BV induces the release of either of histamine or leukotriene $\mathrm{C} 4$ in skin of beekeepers [50], and bvPLA 2 , the major allergen of BV components [51,52], induces a PLA 2 -specific IgE immune responses in mice [53], although BV- and 
bvPLA2-induced Th2 cell immunity and specific IgE production might be protective $[54,55]$. We also observed that a high dose of BV $(2.5 \mathrm{mg} / \mathrm{kg}$, s.c.) treatment could exacerbate oxaliplatin-induced neuropathic pain in rats, whereas low doses of $\mathrm{BV}(0.25$ and $1.0 \mathrm{mg} / \mathrm{kg}$, s.c.) strongly alleviate pain [56]. Thus, the optimal dose and treatment method without side effects should be determined in each disease conditions. Future studies including experimental elucidation of detailed cellular/molecular mechanisms, and well-controlled, randomized clinical trials will lead to a potential therapeutic alternative for treating refractory immunological and neurological diseases.

\section{Acknowledgments}

This work was supported by a grant of the Korea Health Technology R\&D Project through the Korea Health Industry Development Institute (KHIDI), funded by the Ministry of Health and Welfare, Republic of Korea (grant number: HI14 C0738) and by a grant of the National Research Foundation of Korea (NRF) grant funded by the Korea government [MEST] (No. 2012-0005755).

\section{Author Contributions}

H. Bae conceived the idea for the manuscript. D.-S. Hwang and S. K. Kim wrote the manuscript. All three authors revised the manuscript and approved the final manuscript.

\section{Conflicts of Interest}

The authors declare no conflict of interest.

\section{References}

1. Billingham, M.E.; Morley, J.; Hanson, J.M.; Shipolini, R.A.; Vernon, C.A. Letter: An anti-inflammatory peptide from bee venom. Nature 1973, 245, 163-164.

2. Walker, E.W. Bees' stings and rheumatism. Br. Med. J. 1908, 2, 1056-1060.

3. Choi, M.S.; Park, S.; Choi, T.; Lee, G.; Haam, K.K.; Hong, M.C.; Min, B.I.; Bae, H. Bee venom ameliorates ovalbumin induced allergic asthma via modulating $\mathrm{CD} 4{ }^{+} \mathrm{CD} 25^{+}$regulatory $\mathrm{T}$ cells in mice. Cytokine 2013, 61, 256-265.

4. Lee, J.-D.; Park, H.-J.; Chae, Y.; Lim, S. An overview of bee venom acupuncture in the treatment of arthritis. Evid. Based Complement. Altern. Med. 2005, 2, 79-84.

5. Son, D.J.; Lee, J.W.; Lee, Y.H.; Song, H.S.; Lee, C.K.; Hong, J.T. Therapeutic application of anti-arthritis, pain-releasing, and anti-cancer effects of bee venom and its constituent compounds. Pharmacol. Ther. 2007, 115, 246-270.

6. Cho, S.-Y.; Shim, S.-R.; Rhee, H.Y.; Park, H.-J.; Jung, W.-S.; Moon, S.-K.; Park, J.-M.; Ko, C.-N.; Cho, K.-H.; Park, S.-U. Effectiveness of acupuncture and bee venom acupuncture in idiopathic parkinson's disease. Park. Relat. Disord. 2012, 18, 948-952.

7. Kang, S.Y.; Roh, D.H.; Yoon, S.Y.; Moon, J.Y.; Kim, H.W.; Lee, H.J.; Beitz, A.J.; Lee, J.H. Repetitive treatment with diluted bee venom reduces neuropathic pain via potentiation of locus coeruleus noradrenergic neuronal activity and modulation of spinal nrl phosphorylation in rats. J. Pain 2012, 13, 155-166. 
8. Lee, H.; Lee, E.J.; Kim, H.; Lee, G.; Um, E.J.; Kim, Y.; Lee, B.Y.; Bae, H. Bee venom-associated Th1/Th2 immunoglobulin class switching results in immune tolerance of NZB/W F1 murine lupus nephritis. Am. J. Nephrol. 2011, 34, 163-172.

9. Yang, E.J.; Jiang, J.H.; Lee, S.M.; Yang, S.C.; Hwang, H.S.; Lee, M.S.; Choi, S.-M. Bee venom attenuates neuroinflammatory events and extends survival in amyotrophic lateral sclerosis models. J. Neuroinflamm. 2010, 7, 69, doi:10.1186/1742-2094-7-69.

10. Ozdemir, C.; Kucuksezer, U.; Akdis, M.; Akdis, C. Mechanisms of immunotherapy to wasp and bee venom. Clin. Exp. Allergy 2011, 41, 1226-1234.

11. Palomares, O.; Yaman, G.; Azkur, A.K.; Akkoc, T.; Akdis, M.; Akdis, C.A. Role of treg in immune regulation of allergic diseases. Eur. J. Immunol. 2010, 40, 1232-1240.

12. Larché, M.; Akdis, C.A.; Valenta, R. Immunological mechanisms of allergen-specific immunotherapy. Nat. Rev. Immunol. 2006, 6, 761-771.

13. Magnan, A.; Marin, V.; Mely, L.; Birnbaum, J.; Romanet, S.; Bongrand, P.; Vervloet, D. Venom immunotherapy induces monocyte activation. Clin. Exp. Allergy 2001, 31, 1303-1309.

14. Akdis, M.; Schmidt-Weber, C.; Jutel, M.; Akdis, C.A.; Blaser, K. Mechanisms of allergen immunotherapy. Clin. Exp. Allergy Rev. 2004, 4, 56-60.

15. Nam, S.; Ko, E.; Park, S.K.; Ko, S.; Jun, C.Y.; Shin, M.K.; Hong, M.C.; Bae, H. Bee venom modulates murine Th1/Th2 lineage development. Int. Immunopharmacol. 2005, 5, 1406-1414.

16. Sakaguchi, S.; Ono, M.; Setoguchi, R.; Yagi, H.; Hori, S.; Fehervari, Z.; Shimizu, J.; Takahashi, T.; Nomura, T. Foxp $3^{+} \mathrm{CD} 25^{+} \mathrm{CD} 4^{+}$natural regulatory $\mathrm{T}$ cells in dominant self-tolerance and autoimmune disease. Immunol. Rev. 2006, 212, 8-27.

17. Vignali, D.A.; Collison, L.W.; Workman, C.J. How regulatory T cells work. Nat. Rev. Immunol. 2008, 8, 523-532.

18. Bilò, M.B.; Antonicelli, L.; Bonifazi, F. Honeybee venom immunotherapy: Certainties and pitfalls. Immunotherapy 2012, 4, 1153-1166.

19. Cabrera, C.M.; Urra, J.M.; Alfaya, T.; de la Roca, F.; Feo-Brito, F. Expression of Th1, Th2, lymphocyte trafficking and activation markers on $\mathrm{CD}^{+} \mathrm{T}$ cells of hymenoptera allergic subjects and after venom immunotherapy. Mol. Immunol. 2014, 62, 178-185.

20. Mustafa, F.; Ng, F.; Nguyen, T.H.; Lim, L. Honeybee venom secretory phospholipase A2 induces leukotriene production but not histamine release from human basophils. Clin. Exp. Immunol. 2008, 151, 94-100.

21. Martínez-Gómez, J.M.; Johansen, P.; Erdmann, I.; Senti, G.; Crameri, R.; Kündig, T.M. Intralymphatic injections as a new administration route for allergen-specific immunotherapy. Int. Arch. Allergy Immunol. 2008, 150, 59-65.

22. Lee, G.; Bae, H. Phospholipase A2 from bee venom, a novel Foxp $3^{+}$regulatory $\mathrm{T}$ cell inducer, suppresses immune disorders. J. Immunol. 2015, Under Revision.

23. Alunno, A.; Manetti, M.; Caterbi, S.; Ibba-Manneschi, L.; Bistoni, O.; Bartoloni, E.; Valentini, V.; Terenzi, R.; Gerli, R. Altered immunoregulation in rheumatoid arthritis: The role of regulatory T cells and proinflammatory Th17 cells and therapeutic implications. Med. Inflamm. 2015, doi:10.1155/2015/751793.

24. McInnes, I.B.; Schett, G. The pathogenesis of rheumatoid arthritis. N. Engl. J. Med. 2011, 365, 2205-2219. 
25. Kwon, Y.-B.; Lee, J.-D.; Lee, H.-J.; Han, H.-J.; Mar, W.-C.; Kang, S.-K.; Beitz, A.J.; Lee, J.-H. Bee venom injection into an acupuncture point reduces arthritis associated edema and nociceptive responses. Pain 2001, 90, 271-280.

26. Liu, X.; Zhang, J.; Zheng, H.; Liu, F.; Chen, Y. Clinical randomized study of bee-sting therapy for rheumatoid arthritis. Zhen Ci Yan Jiu 2008, 33, 197-200.

27. Jang, H.S.; Kim, S.K.; Han, J.B.; Ahn, H.J.; Bae, H.; Min, B.I. Effects of bee venom on the proinflammatory responses in RAW264.7 macrophage cell line. J. Ethnopharmacol. 2005, 99, 157-160.

28. Jang, H.-S.; Chung, H.-S.; Ko, E.; Shin, J.-S.; Shin, M.-K.; Hong, M.-C.; Kim, Y.; Min, B.-I.; $\mathrm{Bae}, \mathrm{H}$. Microarray analysis of gene expression profiles in response to treatment with bee venom in lipopolysaccharide activated RAW264.7 cells. J. Ethnopharmacol. 2009, 121, 213-220.

29. Park, H.J.; Lee, H.J.; Choi, M.S.; Son, D.J.; Song, H.S.; Song, M.J.; Lee, J.M.; Han, S.B.; Kim, Y.; Hong, J.T. JNK pathway is involved in the inhibition of inflammatory target gene

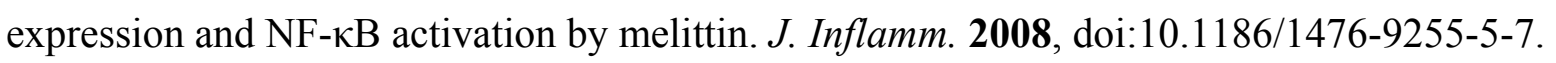

30. Darwish, S.F.; El-Bakly, W.M.; Arafa, H.M.; El-Demerdash, E. Targeting TNF- $\alpha$ and NF-кB activation by bee venom: Role in suppressing adjuvant induced arthritis and methotrexate hepatotoxicity in rats. PLOS ONE 2013, 8, e79284.

31. Agrawal, N.; Chiang, L.K.; Rifkin, I.R. Lupus nephritis. Seminars Nephrol. 2006, 26, 95-104.

32. Mason, L.J.; Berden, J.H. Pathogenic factors for the development of lupus nephritis. Lupus 2008, 17, 251-255.

33. Foster, M.H. Relevance of systemic lupus erythematosus nephritis animal models to human disease. Seminars Nephrol. 1999, 19, 12-24.

34. McFarland, H.F.; Martin, R. Multiple sclerosis: A complicated picture of autoimmunity. Nat. Immunol. 2007, 8, 913-919.

35. Goverman, J. Autoimmune $\mathrm{T}$ cell responses in the central nervous system. Nat. Rev. Immunol. 2009, 9, 393-407.

36. Lee, G.; Lee, H.; Park, S.; Jang, H.; Bae, H. Bee venom attenuates experimental autoimmune encephalomyelitis through direct effects on $\mathrm{CD} 4^{+} \mathrm{CD} 25^{+} \mathrm{Foxp}^{+} \mathrm{T}$ cells. Eur. J. Inflamm. 2013, 11, 111-121.

37. Lee, M.J.; Jang, M.; Choi, J.; Lee, G.; Min, H.J.; Chung, W.S.; Kim, J.I.; Jee, Y.; Chae, Y.; Kim, S.H.; et al. Bee venom acupuncture alleviates experimental autoimmune encephalomyelitis by upregulating regulatory $\mathrm{T}$ cells and suppressing Th1 and Th17 responses. Mol. Neurobiol. 2015, doi:10.1007/s12035-014-9012-2.

38. Paulus, W.; Jellinger, K. The neuropathologic basis of different clinical subgroups of parkinson's disease. J. Neuropathol. Exp. Neurol. 1991, 50, 743-755.

39. Hirsch, E.C.; Breidert, T.; Rousselet, E.; Hunot, S.; Hartmann, A.; Michel, P.P. The role of glial reaction and inflammation in parkinson's disease. Ann. N. Y. Acad. Sci. 2003, 991, 214-228.

40. Block, M.L.; Hong, J.S. Microglia and inflammation-mediated neurodegeneration: Multiple triggers with a common mechanism. Prog. Neurobiol. 2005, 76, 77-98.

41. Kim, J.-I.; Yang, E.J.; Lee, M.S.; Kim, Y.-S.; Huh, Y.; Cho, I.-H.; Kang, S.; Koh, H.-K. Bee venom reduces neuroinflammation in the mptp-induced model of parkinson's disease. Int. J. Neurosci. 2011, 121, 209-217. 
42. Chung, E.S.; Kim, H.; Lee, G.; Park, S.; Kim, H.; Bae, H. Neuro-protective effects of bee venom by suppression of neuroinflammatory responses in a mouse model of parkinson's disease: Role of regulatory T cells. Brain Behav. Immun. 2012, 26, 1322-1330.

43. Alvarez-Fischer, D.; Noelker, C.; Vulinović, F.; Grünewald, A.; Chevarin, C.; Klein, C.; Oertel, W.H.; Hirsch, E.C.; Michel, P.P.; Hartmann, A. Bee venom and its component apamin as neuroprotective agents in a parkinson disease mouse model. PLoS ONE 2013, 8, e61700.

44. Lee, S.-H.; Choi, S.-M.; Yang, E.J. Melittin ameliorates the inflammation of organs in an amyotrophic lateral sclerosis animal model. Exp. Neurobiol. 2014, 23, 86-92.

45. Lee, S.M.; Yang, E.J.; Choi, S.-M.; Kim, S.H.; Baek, M.G.; Jiang, J.H. Effects of bee venom on glutamate-induced toxicity in neuronal and glial cells. Evid. Based Complement. Altern. Med. 2011, 2012, doi:10.1155/2012/368196.

46. Kim, S.K.; Bae, H. Acupuncture and immune modulation. Auton. Neurosci. 2010, 157, 38-41.

47. Maddur, M.S.; Miossec, P.; Kaveri, S.V.; Bayry, J. Th17 cells: Biology, pathogenesis of autoimmune and inflammatory diseases, and therapeutic strategies. Am. J. Pathol. 2012, 181, 8-18.

48. Kim, H.; Lee, G.; Park, S.; Chung, H.S.; Lee, H.; Kim, J.Y.; Nam, S.; Kim, S.K.; Bae, H. Bee venom mitigates cisplatin-induced nephrotoxicity by regulating $\mathrm{CD} 4{ }^{+} \mathrm{CD} 25^{+} \mathrm{Foxp} 3^{+}$regulatory T-cells in mice. Evid. Based Complement. Altern. Med. 2013, doi:10.1155/2013/879845.

49. Chen, J.; Lariviere, W.R. The nociceptive and anti-nociceptive effects of bee venom injection and therapy: A double-edged sword. Prog. Neurobiol. 2010, 92, 151-183.

50. Annila, I.; Saarinen, J.V.; Nieminen, M.M.; Moilanen, E.; Hahtola, P.; Harvima, I.T. Bee venom induces high histamine or high leukotriene $\mathrm{c} 4$ release in skin of sensitized beekeepers. J. Investig. Allergol. Clin. Immunol. 2000, 10, 223-228.

51. Annila, I. Bee venom allergy. Clin. Exp. Allergy 2000, 30, 1682-1687.

52. Sobotka, A.K.; Franklin, R.M.; Adkinson, N.F.; Valentine, M.; Baer, H.; Lichtenstein, L.M. Allergy to insect stings: II. Phospholipase A: The major allergen in honeybee venom. J. Allergy Clin. Immunol. 1976, 57, 29-40.

53. Dudler, T.; Machado, D.C.; Kolbe, L.; Annand, R.R.; Rhodes, N.; Gelb, M.H.; Koelsch, K.; Suter, M.; Helm, B.A. A link between catalytic activity, IgE-independent mast cell activation, and allergenicity of bee venom phospholipase A2. J. Immunol. 1995, 155, 2605-2613.

54. Marichal, T.; Starkl, P.; Reber, L.L.; Kalesnikoff, J.; Oettgen, H.C.; Tsai, M.; Metz, M.; Galli, S.J. A beneficial role for immunoglobulin $\mathrm{E}$ in host defense against honeybee venom. Immunity 2013, 39, 963-975.

55. Palm, N.W.; Rosenstein, R.K.; Yu, S.; Schenten, D.D.; Florsheim, E.; Medzhitov, R. Bee venom phospholipase A2 induces a primary type 2 response that is dependent on the receptor ST2 and confers protective immunity. Immunity 2013, 39, 976-985.

56. Lim, B.S.; Moon, H.J.; Li, D.X.; Gil, M.; Min, J.K.; Lee, G.; Bae, H.; Kim, S.K.; Min, B.I. Effect of bee venom acupuncture on oxaliplatin-induced cold allodynia in rats. Evid. Based Complement. Altern. Med. 2013, doi:10.1155/2013/369324.

(C) 2015 by the authors; licensee MDPI, Basel, Switzerland. This article is an open access article distributed under the terms and conditions of the Creative Commons Attribution license (http://creativecommons.org/licenses/by/4.0/). 